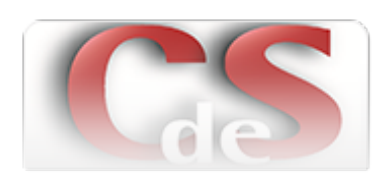

Cuestiones de sociología ISSN: 2346-8904

publicaciones@fahce.unlp.edu.ar

Universidad Nacional de La Plata

Argentina

\title{
La salud y la enfermedad como problema social
}

Pagnamento, Licia; Castrillo, Belén; Hasicic, Cintia

La salud y la enfermedad como problema social

Cuestiones de sociología, núm. 22, 2020

Universidad Nacional de La Plata, Argentina

DOI: https://doi.org/10.24215/23468904e089

Atribución no comercial compartir igual (CC BY-NC-SA) 4.0 
Presentación

\section{La salud y la enfermedad como problema social}

Health and disease as a social problem

Licia Pagnamento

DOI: https://doi.org/10.24215/23468904e089

Centro Interdisciplinario de Metodología en Ciencias

Sociales, Instituto de Investigaciones en Humanidades y

Ciencias Sociales, Facultad de Humanidades y Ciencias

de la Educación, Universidad Nacional de La Plata,

Argentina

pagnamento@yahoo.com.ar

\section{Belén Castrillo}

Centro Interdisciplinario de Metodología en Ciencias

Sociales, Instituto de Investigaciones en Humanidades y

Ciencias Sociales, Facultad de Humanidades y Ciencias

de la Educación, Universidad Nacional de La Plata,

Argentina

mbcastrillo@gmail.com

\section{Cintia Hasicic}

Instituto de Investigaciones Gino Germani, Facultad de

Ciencias Sociales, Universidad de Buenos Aires, Argentina

cintiahasicic@yahoo.com.ar

\section{RESUMEN:}

A modo de presentación del dossier, este artículo expone una breve reflexión sobre los alcances de lo que podemos denominar como Sociología de la salud, colocándonos frente a un conjunto de temas diversos que ofrecen la oportunidad de discutir o resituar en el debate público ciertos problemas asociados a la salud, enfermedad, atención y cuidados, al inscribirlos en el marco de la interpretación/explicación sociológica. La diversidad de temas y problemas reciben en este dossier una aproximación, inicial y no exhaustiva, que invita a recorrer aportes y discusiones de relevancia para la salud colectiva.

PALABRAS CLAVE: Sociología y salud, Investigaciones actuales en salud, Medicina social latinoamericana.

\section{AbStraCt:}

As a presentation of the dossier, this article presents a brief reflection on the scope of what we call Health Sociology, facing a set of diverse issues that offer the opportunity to discuss or resituate certain associated problems in the public debate to health, illness and care, by enrolling them in the framework of the sociological interpretation/explanation The diversity of issues and problems receive in this dossier an initial and non-exhaustive approach, which invites us to explore contributions and discussions of relevance to collective health.

KEYWORDS: Sociology and health, Current health research.

¿Existe una sociología sobre la salud y la enfermedad? La pregunta entre curiosa e incrédula deja abierta la entrada a un problema social que suele reservarse a la medicina u otro tipo de conocimiento experto. Sin embargo, lo social emerge como una dimensión central frente a aquello que nuestras sociedades consideran como salud, enfermedad o padecimientos. Este interrogante puede ser interpretado entonces como un disparador de esta reflexión inicial que introduce el tema desarrollado en el dossier. 
Empecemos por trazar las lábiles fronteras de un área de reflexión compartida por varias ciencias sociales que ha demostrado un temprano interés en ciertos fenómenos vinculados a la salud y la enfermedad. En particular, en sociología no será sorpresa encontrarnos con las muy conocidas apreciaciones de Parsons sobre el rol del médico y del enfermo desplegadas en el capítulo décimo de "El sistema social" (1951), o el análisis de Goffman (1972) sobre las instituciones psiquiátricas. También se suele referir el estudio de Durkheim sobre el suicidio (obra de 1897), un autor que supo explorar en la oposición normal/patológico, una metáfora para pensar el orden social. Por supuesto la preocupación central en estos casos descansaba más en ofrecer una demostración clarificadora sobre aserciones más o menos abstractas acerca de la sociedad que en la delimitación de un área de indagación con relativa independencia.

Este es un pequeño recorte que permite rememorar autores y obras ampliamente conocidas, y ofrece un primer acercamiento a lo que podríamos llamar ciertos temas de interés relativos a la salud, la enfermedad y las respuestas construidas por los/as sujetos/as y/o los conjuntos sociales frente a ellas. Pero aún antes de la época en que Durkheim se detuviera a construir como problema social uno que solía asociarse más bien a cierto tipo de enfermedad o patología, transcurrían discusiones en un ámbito más amplio que el de la sociología. Esas discusiones planeaban sobre la profusión y el aumento de ciertas enfermedades, sobre el sufrimiento y la muerte, o las desviaciones moralmente reprochables desde la perspectiva de la burguesía en ascenso hacia prácticas sociales identificadas en la clase obrera (ejemplos paradigmáticos son el consumo de alcohol, la prostitución o la "promiscuidad"). En ese contexto emergió uno de los registros más descarnados sobre la vinculación entre ciertas enfermedades/problemas de salud y las condiciones de vida y trabajo en la clase obrera, provisto por Engels (1845). Aunque no fuera el objetivo central de su trabajo, la situación de la salud, el deterioro/desgaste físico y las enfermedades en la clase obrera, era un tema insoslayable al caracterizar la vida del proletariado en la Inglaterra del siglo XIX, además de formar parte de las propias demandas y estrategias de organización de la propia clase, así como de las más tempranas respuestas por parte del Estado (particularmente en Inglaterra) y el intento de la burguesía por controlar el conflicto social.

Por otra parte, la sociología no ha sido el único cuerpo de conocimiento científico que posó su mirada en este tipo de fenómenos. Los/las antropólogos/as, desde los inicios de la conformación de la disciplina, acercaban relatos sobre las prácticas médicas y las creencias sobre los daños a la salud en sociedades no occidentales (Lieban, 1977). Ese temprano interés se consolidó y conformaría, hasta la actualidad, una de las ramas u orientaciones de investigación más conocidas de esa disciplina.

Asimismo, en el siglo XIX el enfoque de la medicina social del continente europeo se había desarrollado de la mano de médicos como Virchow en Alemania o Villerme en Francia, Farr en Inglaterra y Puccinotti en Italia. Ellos enfatizaban el carácter de ciencia social de la medicina y discutían sobre la necesidad de que la sociedad/el Estado asegurara la salud de sus integrantes (García, 1989). Esta forma de entender la imbricación entre la medicina y las ciencias sociales estuvo presente en los debates académicos al menos hasta la primera parte del siglo XX, período en que progresivamente se conformó una perspectiva alternativa sobre la enfermedad y la medicina que desplazó los componentes sociales a favor de lo biológico como eje central de explicación de la enfermedad y de una concepción mecanicista del hombre (Navarro, 1982; Berliner, 1988).

Ya entrado el siglo XX y especialmente en la década de los años 60, en los países centrales tanto como en los dependientes emergió un conjunto de cuestionamientos en torno a diversas facetas y características de la medicina moderna. Entre ellas se ubican los crecientes costos de la atención, el aumento del consumo/ prescripción de fármacos e intervenciones médicas en problemas que anteriormente no las requerían, los límites a la eficacia médica y sus más evidentes fracasos (alcoholismo, drogadicción, etc.), el incremento de la iatrogenia negativa, entre otros. Por su parte, América Latina agrega a estos cuestionamientos problemas específicos de su región como la persistencia de muertes evitables, problemas de infraestructura básica, hambre y desnutrición, crisis de financiamiento de los sectores prestadores de servicios de salud, entre otros (Menéndez, 1990). 
Es justamente en este particular contexto que emerge una corriente intelectual que recupera la pregunta por la relación entre la sociedad y los problemas de salud colectivos. A partir de allí, puede señalarse que Latinoamérica perfila una rica tradición intelectual que articula conocimientos de las ciencias sociales y las ciencias médicas (especialmente entre estas últimas aquellas orientaciones preocupadas por el abordaje de la dimensión social). Como recuerda Castro (2011), esta tradición conocida como medicina social latinoamericana, se remonta a la década de los sesenta y cuenta con una prolífica producción y enfoques diversos. Conviene aclarar que esta denominación sortea el problema de las múltiples designaciones (medicina social, ciencias sociales aplicadas a la salud, entre otras) destinadas a un conjunto de trabajos sobre temas de salud y enfermedad en los que lo social ocupa un lugar destacado. Esta corriente configuró un ámbito de reflexión multidisciplinar que continúa siendo un punto de referencia aun cuando en las últimas décadas ha perdido el impulso y dinamismo que detentara en sus orígenes. Aun reconociendo las variaciones al interior de esta corriente, su legado más conocido radica en que, partiendo del materialismo histórico, propuso no solo un marco conceptual riguroso sino también la discusión sobre la construcción del propio objeto de estudio (¿"salud”? ¿ “enfermedad”?), la necesidad de su redefinición discutiendo la aproximación de la biomedicina repensando los conceptos más apropiados para abordarlo, y consecuentemente la o las estrategias metodológicas más apropiadas para abordarlo. Aún a riesgo de una mención reductiva, puede indicarse que Asa Cristina Laurell y Eduardo Menéndez son quizás dos de los más conocidos representantes de las discusiones conceptuales a las que hacemos referencia, aportando los conceptos de proceso saludenfermedad (Laurell, 1982), proceso salud-enfermedad-atención y modelo médico hegemónico (Menéndez, 1990). Junto a ellos, se ubican también las aproximaciones de J. Frenk (1985), R. B. Mendes Goncalves (1979), V. Navarro (1982) y Juan César García (García, JC., 1981; Galeano y Colab, 2011), quienes exploraron las categorías de trabajo, clase social, trabajo médico, analizaron la organización de la prestación del servicio de salud y plantearon la necesidad de reflexionar sobre la definición de los problemas que se incluyen en el área.

Como señala Castro (2011), a pesar de todo el conocimiento que se ha generado, quienes provienen de la salud pública y la medicina social no suelen estar familiarizados con la diversidad teórica (y metodológica) dentro de las ciencias sociales ni con sus aportes al campo de la salud. De manera similar, los/as estudiantes de ciencias sociales no siempre tienen en sus horizontes la construcción de la salud/la enfermedad como objeto de trabajo de sus disciplinas.

Sin embargo, las preocupaciones intelectuales por generar explicaciones sobre, o frente a, este tipo de problemas siguen reactualizándose. Resulta significativo también que, al menos potencialmente, traspasan las fronteras disciplinares, de la misma manera que seguramente exceden las fronteras del conocimiento científico al formar parte de aproximaciones y prácticas de los propios conjuntos sociales.

Visto de esta manera, el reencuentro y la posibilidad de abordar problemas referidos a la salud y la enfermedad, reeditan el hecho de que forman parte de los problemas de carácter social que afectan a distintos conjuntos sociales contextuados en términos históricos. La salud y la enfermedad están involucradas en las condiciones de vida, la desocupación, la precarización laboral, las condiciones de trabajo adversas que afectan la salud de los/as trabajadores/as, la orientación político-ideológica de la educación de las generaciones más jóvenes, la atención médica respetuosa de los derechos de las personas, las características del sistema de salud con sus alcances, limitaciones, contradicciones y problemas. Tomando la propuesta de Castel (2006), estas y otras configuraciones problemáticas constituyen un programa privilegiado de investigación (tanto teórica como práctica) en sociología, y permiten recuperar demandas sociales que se construyen en el entramado de conflictos y contradicciones propios de la sociedad moderna.

En sociología, se cuenta con herramientas conceptuales y metodológicas propias, así como la posibilidad de explorar aquellas provenientes de disciplinas afines (pensemos por ejemplo en la antropología o la historia) dado que el conjunto de las ciencias sociales han desplegado un recorrido vasto, heterogéneo y fructífero. Podría argumentarse también (y discutirse) la persistencia de interrogantes típicos de la disciplina, o su 
“perspectiva cognitiva” (Bauman y May, 2007), conformada por el grupo de categorías, interrogantes, problemáticas, y respuestas provisorias y alternativas propias de la pluralidad teórica y metodológica de la sociología.

Finalmente se debe reconocer que quienes se acercan a temas referidos a la salud, la enfermedad, los padecimientos (es decir aquellos fenómenos y situaciones que la biomedicina hegemónica no define como enfermedad pero que son percibidos como malestar/es por los/as sujetos/as), y las prácticas sociales orientadas a brindarles algún tipo de respuesta, enfrentan el desafío de construir una aproximación sociológica a problemas que cuentan con respuestas previas, no sólo atinentes al sentido común, sino a la aproximación ampliamente aceptada de la medicina (que forma parte, además, del sentido común).

En esta dirección, el punto de inicio (la ruptura con el sentido común) nos ubica muchas veces en una posición desafiante frente a saberes que no suelen ser cuestionados y que detentan una posición de poder significativa. Lógicamente las reacciones frente a los resultados de las investigaciones desde la perspectiva de la sociología pueden ser airadas y controversiales. Sin embargo, los estudios sólidamente fundados (en términos teórico-metodológicos) ofrecen elementos de importancia no sólo por el desarrollo del conocimiento, sino por su vinculación con problemas que no pueden quedar apartados del debate público. Este último punto es especialmente delicado en los temas de salud porque competen a un conjunto de problemas sociales que al quedar envueltos en una mirada técnica suelen ser retirados de dicho debate, detrás de un pretendido embozo de neutralidad y objetividad médica.

Volviendo sobre la idea de que la salud y la enfermedad constituyen "configuraciones problemáticas" y que por lo tanto conforman o delimitan un programa de investigación privilegiado (Castel, 2006), la diversidad de temas abordados, aun conociendo el hecho de que los problemas de investigación se estructuran en torno a algunos que concentran la atención en ciertos momentos en particular, es una buena manera de caracterizar los intereses de los/as investigadores/as que contribuyen (entre otras cuestiones) a resituar problemas sociales en el debate público.

Una forma de hacer visible la diversidad y a la vez los temas más abordados, se refleja en los artículos que componen este dossier, aun cuando el panorama que tracemos sea incompleto.

Pero también quisiéramos mencionar la producción y contribución de nuestros/as estudiantes de sociología de la FaHCE-UNLP que optan por escribir sus trabajos finales de licenciatura (y este interés no sólo emerge por/al transitar espacios curriculares dedicados específicamente al tema) sobre temas vinculados a salud. Si nos concentramos en los temas de los trabajos (30 presentados entre 2003 y 2019) una de las áreas de interés que emerge claramente compete a la salud sexual reproductiva y no-reproductiva y las relaciones de género vinculadas a la salud, sobre todo en los últimos años (particularmente desde 2011). Pueden agruparse aquí trabajos que abordan problemáticas sobre los derechos sexuales y reproductivos en relación a instituciones/programas de salud y educación; masculinidades y su relación con la salud sexual; maternidades/paternidades jóvenes en sectores vulnerables; partos planificados en domicilios; aborto; modificaciones corporales travestis y su relación a la salud-enfermedad-atención. ${ }^{1}$

Otro grupo de tesinas indagan sobre procesos relacionados con las políticas de salud, recalan en el funcionamiento del propio sector salud, y de políticas referidas a temas de salud que no necesariamente implementa dicho sector. Los trabajos toman como foco de reflexión a las instituciones prestadoras de servicios de salud y recortan en ese marco un conjunto diverso de problemáticas: condiciones/características de la provisión de servicios, recursos, organización de actividades y objetivos. Otros, analizan políticas en el ámbito de la salud considerando las actividades que concretamente se despliegan y, finalmente, el análisis de discusiones en torno a marcos normativos que se relacionan directamente con condiciones de salud de la población (por ejemplo la discusión referida a la obesidad). ${ }^{2}$

Un tercer conjunto de tesinas se centra en la práctica biomédica (ejercicio profesional y formación de los/as profesionales de la salud), la perspectiva médica sobre la adherencia a tratamientos médicos en ciertas enfermedades crónicas, perspectivas sobre las adicciones, las articulaciones entre distintas prácticas médicas 
para enfrentar enfermedades/padecimientos, las relaciones entre la biomedicina y prácticas alternativas y las experiencias de atención/abordaje de problemas de salud que se presentan como alternativos. Este conjunto de trabajos con temas variados, puede caracterizarse (aun cuando no los englobe a todos) como estudios referidos a las prácticas médicas tanto biomédicas como alternativas. ${ }^{3}$

Finalmente, aun cuando sean sólo tres, está presente la reflexión en torno al trabajo y a la categoría de trabajo, ambas vinculadas a la salud. Aquí ubicamos un trabajo que propone repensar algunos problemas de salud mental en relación a la categoría de trabajo humano. También disponemos dedos producciones que reflexionan sobre las condiciones de trabajo/salud en trabajadores/as de la salud y de la educación. ${ }^{4}$

Las tesinas tomadas como referencia parten de un cuerpo conceptual diverso, no necesariamente inscripto en la corriente de la medicina social latinoamericana que se mencionó al inicio, pero poniendo en juego herramientas teóricas propias de la sociología. Suman, además, aproximaciones de disciplinas afines y/o discusiones en boga que en el campo de la salud incluyen aquellas que se desenvuelven en torno a documentos de organismos internacionales como la OPS/OMS o UNICEF, por nombrar a dos de los más conocidos, cuyos contenidos invitan a discusiones conceptuales (muchos informes recogen perspectivas provenientes de las ciencias sociales) y a interpretar y analizar marcos legales, normas y protocolos de intervención como expresión o referencia a prácticas y representaciones sociales en torno a la salud y la enfermedad.

En resumidas cuentas, los trabajos de investigación de los/as jóvenes sociólogos/as que mencionamos aquí, revelan el interés por reflexionar sobre problemas de relevancia social relacionados con la salud y la enfermedad. Un interés que forma parte de la construcción del oficio en términos colectivos. Esto puede ser pensado así, si se recupera la analogía de R. Ortiz (2004) sobre el oficio intelectual del científico social (se requiere de habilidades y cierto conocimiento, y es con la práctica acumulada a lo largo de los años, que se logra producir un objeto). Parte de este oficio, si se lo piensa como producto colectivo (y no meramente individual), se construye en los ejercicios de investigación que permiten concluir los estudios de grado y constituyen un aporte al área o el campo de reflexión sobre la salud y la enfermedad. No se trata de una mera acumulación de productos individuales, sino más bien un área de reflexión en construcción en la que los primeros pasos en investigación ofrecen senderos que invitan a ser recorridos, citados, revisados, apropiados por otros/as.

En la diversidad de las "configuraciones problemáticas" vinculadas a la salud y la enfermedad, el recorrido de este dossier recupera algunos temas centrales en los debates actuales. Presenta entonces algunos nodos temáticos en torno a las políticas de protección social y salud, las relaciones de poder y asimetría que afectan la salud/bienestar de las personas, el imperativo de la salud en nuestras sociedades, los problemas de salud laboral. Por otra parte el dossier está atravesado por el debate actual sobre género y derechos humanos, que toman especial visibilidad en algunos temas y problemas como el aborto, el abuso sexual o los partos.

En la sección Artículos encontramos un trabajo de Liliana Findling, Elsa López y Estefanía Cirino que traza un panorama sobre las personas mayores en el Gran Buenos Aires, recorriendo conceptos teóricos referidos al envejecimiento y las políticas sociales así como a las desigualdades en salud. Simultáneamente, dicho artículo se propone describir los cambios ocurridos en las políticas de protección social desde 2015 hasta la actualidad y analizar datos sobre previsión social y acceso a la salud.

Romina Del Mónaco presenta un trabajo de resuelta originalidad en el área, al enfocar ciertas prácticas profesionales asociadas a procesos terapéuticos en el terreno de la psicología. Propone explorar las terapias cognitivo-conductuales en la Ciudad Autónoma de Buenos Aires, recurriendo a las narrativas de profesionales entrevistados/as para analizar el lugar de la "brevedad" como un aspecto que atraviesa y modela el proceso terapéutico.

Alejandro Capriati, Gabriela Wald y Ana Clara Camarotti reflexionan sobre el abuso sexual entendiéndolo como una violación de los derechos humanos (que afecta el bienestar físico, sexual, reproductivo, emocional, mental y social de las personas y las familias que lo padecen) y que se imbrica en jerarquías de género y abusos de autoridad. Proponen un modelo de intervención comunitario e integral para 
prevenir y dar respuesta a situaciones de abuso sexual infantoadolescente, en base a experiencias de trabajo previas.

En la sección Debate del dossier, se incluye un trabajo de Josefina Brown que analiza un tema de especial actualidad, en el que rastrea los clivajes de la construcción de aborto como un problema social y como un derecho en la historia argentina reciente (1983-2018).

Este tema se relaciona estrechamente con una de las notas de investigación realizada por Hernán Cáneva que, en torno a una relectura de su trabajo de tesis doctoral sobre las representaciones sociales del aborto, invita a reflexionar sobre la instancia de divulgación científica. Una instancia que reconocemos como clave en el proceso de reubicación de la salud colectiva en el debate público.

La segunda nota de investigación que se incluye en el dossier, escrita por Belén Castrillo, recupera sus resultados de investigación doctoral vinculada a la atención médica de embarazos y partos. Una temática que ha recibido mayor atención, a partir de las discusiones sobre violencia obstétrica, y que la autora enmarca en procesos y representaciones sociales mayores, interpelados desde la consideración de los derechos humanos y género.

Finalmente, la tercera nota de investigación realizada por Laura Henry y Sofía Malleville se ubica en la intersección de la sociología de la salud y la sociología del trabajo, y presenta una indagación empírica sobre los riesgos psicosociales asociados al trabajo a partir de un análisis de caso. Se trata de un aporte que suma al conjunto de los debates actuales un tema (aún con las diferencias del caso) que ha sido desplazado por otros en las últimas décadas y que fuera objeto de intensa reflexión en el último tramo del siglo pasado.

Forma parte de este dossier una entrevista al destacado sociólogo mexicano Roberto Castro Pérez, cuyos aportes a la sociología de la salud incluyen su conocida apropiación de la teoría bourdiana aplicada a la reflexión sobre temas relacionados con la salud colectiva. Sus trabajos de investigación recorren tópicos referidos a los determinantes sociales de la experiencia subjetiva de la salud, al ejercicio de diferentes violencias (contra las mujeres, en el noviazgo y contra la infancia en México) y a la violación de los derechos reproductivos de las mujeres en los servicios de salud.

Finalmente se presentan tres reseñas de obras actuales que problematizan el imperativo moral de la salud (Coniglio), las condiciones de vida de la población trans (Romero Marchesini) y las prácticas y participación sindical en el ámbito del hospital público (Malleville).

\section{ReFERENCiAS}

Bauman, Z. y May, T. (2007). Pensando sociológicamente. Buenos Aires: Nueva Visión.

Berliner, H. (1988). Una perspectiva más amplia sobre el informe Flexner. Presentado en Segundas Jornadas de Atención Primaria de la salud. Buenos Aires.

Castel, R. (2006). La sociología y la respuesta a la demanda social. En B. Lahire, ¿Para qué sirve la sociología? Buenos Aires: Siglo Veintiuno Editores.

Castro, R. (2011). Teoría Social y Salud. Buenos Aires: Lugar Editorial.

Engels, F. (1976). La situación de la clase obrera en Inglaterra, Vol. 29, Madrid: Akal. Primera edición 1845

Frenk, J. (1985). El concepto y la medición de accesibilidad, Revista de Salud Pública de México, 27 (5): 438-53.

Galeano, D., Trotta, L. y Spinelli, H. (2011). Juan César García y el movimiento latinoamericano de medicina social: notas sobre una trayectoria de vida. Salud Colectiva, 7 (3):285-315.

García, J. C. (1989). Entrevista a Juan César García. Ciencia y Sociedad, 16(1).

García, J. C. (1994). La enfermedad de la pereza. En: Pensamiento social en salud en América Latina. México. Mc Graw Hill, pp. 150-171. Primera edición 1981.

García, J.C. (1983). La categoría trabajo en la medicina. Cuadernos Médico Sociales N²3 pp. 1-11. Rosario, Argentina. 
Goffman, I. (1972). Internados. Ensayos sobre la situación social de los enfermos mentales. Buenos Aires, Amorrortu Editores. Primera edición en inglés, 1961.

Lieban, R. W. (1977). El campo de la antropología médica. Cultura, enfermedad y curación. New York: Macmillan Marradi, A., Archenti, N. y Piovani, J. (2007). Metodología de las ciencias sociales. Buenos Aires: Emecé.

Mendes Goncalves, R. B. (1979). Medicina e historia: raices sociales del trabajo médico. San Pablo: Facultad de Medicina de la Universidad de San Pablo.

Menéndez, E. (1990). Morir de alcohol. Saber y hegemonía médica. México: Alianza Editorial.

Navarro, V. (1982). Trabajo, ideología y ciencia: el caso de la medicina. Revista Latinoamericana de Salud $\mathrm{N}^{\circ} 2$. México.

Ortiz, R. (2004). Taquigrafiando lo social. Buenos Aires: Siglo XXI.

Parsons, T. (1951). The Social System. Londres: Routledge \& Kegan Paul [1979].

\section{Notas}

1 Berenise Martínez (2019), Paula Provenzano (2017), Natalia Romero Merchesini (2016), M. Herminia Maldován (2015), Elisa Pereyra (2014), Vianel Silvana García (2013), Emilce Insaurralde (2013), Hernán Caneva (2012), Cintia Hasicic (2011), Graciela Bellotti (2006). Disponibles en Memoria Académica, FaHCE-UNLP: http://www.memoria .fahce.unlp.edu.ar

2 Manuela Nogueira Expósito (2017), Belén Castrillo (2013), Beliera, Anabel (2011), Natalia García (2009), Bernardo Lacunza (2007), Evangelina Odorizzi (2004), Florencia Demarche (2004), Mariana Specogna (2003), Mercedes Martiarena (2003). Disponibles en Memoria Académica, FaHCE-UNLP: http://www.memoria.fahce.unlp.edu.ar

3 Lupe Marín (2017), Jimena Coniglio (2017), Stimbaum, Camila (2017), María Eugenia Bellocq (2011), Marianela Pinzas (2010), María Inés Mangano (2008), María Azucena Sampayo (2005), Pablo Vetere (2006). Disponibles en Memoria Académica, FaHCE-UNLP: http://www.memoria.fahce.unlp.edu.ar

4 Guadalupe Guaresti (2018), Sofía Malleville (2017), Chaves y Ramella (2010). Disponibles en Memoria Académica, FaHCE-UNLP: http://www.memoria.fahce.unlp.edu.ar

\section{BY-NC-SA}

